\title{
FPGA-BASED EFFICIENT HARDWARE/SOFTWARE CO-DESIGN FOR INDUSTRIAL SYSTEMS WITH CONSIDERATION OF OUTPUT SELECTION
}

\author{
Kyriakos M. Deliparaschos ${ }^{*}$ - Konstantinos Michail ${ }^{* *}$ \\ Argyrios C. Zolotas ${ }^{* *}$ - Spyros G. Tzafestas ${ }^{* * *}$
}

\begin{abstract}
This work presents a field programmable gate array (FPGA)-based embedded software platform coupled with a softwarebased plant, forming a hardware-in-the-loop (HIL) that is used to validate a systematic sensor selection framework. The systematic sensor selection framework combines multi-objective optimization, linear-quadratic-Gaussian (LQG)-type control, and the nonlinear model of a maglev suspension. A robustness analysis of the closed-loop is followed (prior to implementation) supporting the appropriateness of the solution under parametric variation. The analysis also shows that quantization is robust under different controller gains. While the LQG controller is implemented on an FPGA, the physical process is realized in a high-level system modeling environment. FPGA technology enables rapid evaluation of the algorithms and test designs under realistic scenarios avoiding heavy time penalty associated with hardware description language (HDL) simulators. The HIL technique facilitates significant speed-up in the required execution time when compared to its software-based counterpart model.
\end{abstract}

K eywords: sensor optimization, FPGA, maglev, embedded control, electromagnetic suspension, linear quadratic Gaussian, hardware-in-the-loop, FPGA-in-the-loop

\section{INTRODUCTION}

Most control systems nowadays are embedded in a sense that they rely on built-in special purpose digital hardware to close any feedback loops. Embedded control systems are widely used in industrial control, transportation systems, robotics, automobiles, aircrafts (incl. UAVs), household appliances and other. These types of system interface with the external environment (ie, sensors and actuators) are real-time critical (ie, embedded control algorithm must execute in synchrony with the physical system which is controlled to guarantee performance and safety), as well as allowing for distributed control (ie, a network of embedded controllers). A modelbased embedded control software/hardware co-design approach is followed in this work. Modeling/simulation alongside FPGA synthesis and HDL analysis tools (ie, MATLAB/Simulink and Xilinx ISE) is used to enable rapid prototyping, autocode generation (ie, generate HDL code from a Simulink model), Hardware-In-theLoop (HIL) testing, and consider the functional correctness of the model-based design with the generated HDL in a co-simulation environment. HIL techniques are widely used in the development and testing of complex real-time control systems by effectively adding the complexity of the plant under control to the test platform $[1,2]$. The model of the system is realized in soft form and modeled via high-level language (eg, MATLAB) or a graphical model-based design tool (eg, Simulink). Figure 1 illustrates an embedded control system, where the model of the plant (realized on software) interfaces with the actual controller (implemented on hardware) via a communication link.

A typical high integrity system requires both control and reliable operation. Optimized performance, robustness, fault tolerance, and low complexity are the main goals of the designer. Industrial plants require a set of sensor nodes for acquiring measurements of the system. Part of this work is focused on minimizing the number of sensors selected from a large set such that the system is (i) stable, and (ii) satisfies a number of closed-loop performance criteria. The task of sensor set selection in an optimized manner for control design is a non-trivial task to do; especially if there is a large number of sensor candidates to select from.

In [3], a framework for control and fault tolerance is proposed, which takes into account the aforementioned requirements for a non-trivial problem: the control of an electromagnetic suspension (EMS) for a maglev train [4]. The systematic framework combines LQG control [5], multi-objective optimization using genetic algorithms (GA) [6], and reconfigurable fault tolerant control methods $[7,8]$. The maglev EMS was used to test the efficacy of the framework and the results, implemented at a sim-

\footnotetext{
* Department of Electrical, Computer Engineering and Informatics, Cyprus University of Technology, Limassol, CY, k.deliparaschos@cut.ac.cy; ${ }^{* *}$ SignalGenerix Ltd, Limassol, CY, kon_michael@ieee.org; *** School of Engineering, College of Science, University of Lincoln, UK, azolotas@lincoln.ac.uk; ${ }^{* * *}$ School of Electrical and Computer Engineering, National Technical University of Athens, GR, tzafesta@softlab.ntua.gr
} 


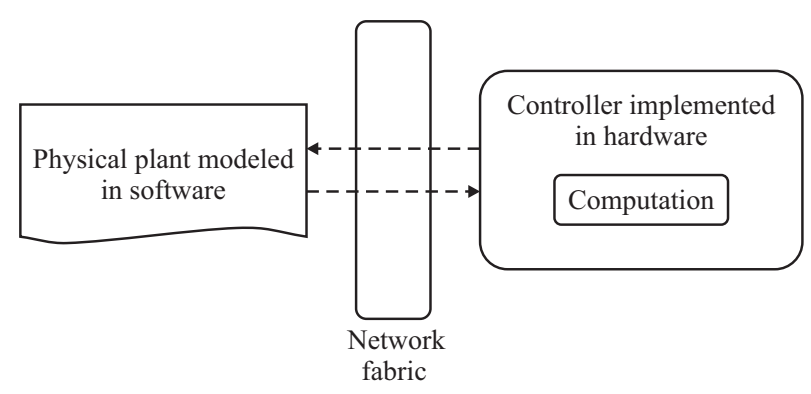

Fig. 1. A simplified diagram of an embedded control system

ulation level only, illustrate good potential for industrial applications.

In this paper, the validation of the framework is done via a model-based embedded control hardware/software co-design approach $[9,10]$, where a full LQG controller (combination of a linear-quadratic-regulator (LQR) and a Kalman-Bucy estimator (KBE)) is implemented on an FPGA chip and a HIL scheme is employed [11] for practical integration of the LQG controller on the FPGA chip [12] with the physical process describing the EMS plant under control, modeled in a high-level simulation environment (MATLAB/Simulink). In this setup, the control of an inherently unstable, nonlinear maglev EMS subject to a set of non-trivial control requirements (that industrial systems of such nature have), using the minimum number of sensors was studied. In [13], the authors presented some initial validation results of the systematic framework using the HIL technique targeted on FPGA, hereinafter referred to as FPGA-in-the-loop (FIL). While the $\mathrm{KBE}$, as part of the LQG controller, was implemented on an FPGA chip, unlike our approach in this paper, the LQR was modeled in MATLAB/Simulink. Moreover, a comparison with increased number of sensor sets was made in terms of performance and FPGA resource requirements. The results show that the performance remains the same even if more sensors are added in the set. Moreover, the FPGA resource requirements are significantly reduced when fewer sensors are used to control the system. Finally, the FIL technique, as expected, shows a significant speed-up in the required execution time when compared to the software-based model.

The rest of this paper is organised as follows. Section 2 outlines the modeling aspects of the maglev EMS system. Section 3 describes the systematic framework for optimized sensor selection with the FIL concept and Section 4 introduces the LQG architecture and implementation on the FPGA. Analysis, discussion on robustness and simulation results from the practical LQG implementation with FIL as applied on the EMS are discussed in Section 5, while Section 6 provides conclusions.

\section{CONTROL REQUIREMENT OF THE EMS MODEL}

The section discusses the maglev model for this study, while also presents the particulars on EMS control speci- fications prior to proceeding with sensor selection and the rest of analysis and implementation steps.

\subsection{Non-linear model of the EMS}

The single-stage EMS that represents one quarter of a typical maglev vehicle, is based on a typical U-core shape electromagnet. The non-linear model is described as follows (for details the reader can refer to [4]),

$$
\begin{aligned}
\frac{\mathrm{d} I}{\mathrm{~d} t} & =\frac{V_{c}-I R_{c}+\frac{N_{c} A_{p} K_{b}}{G^{2}}\left(\frac{\mathrm{d} z_{t}}{\mathrm{~d} t}-\frac{\mathrm{d} Z}{\mathrm{~d} t}\right)}{\frac{N_{c} A_{p} K_{b}}{G}+L_{c}}, \\
B & =K_{b} \frac{I}{G}, \\
F & =K_{f} B^{2}, \\
\frac{\mathrm{d}^{2} Z}{\mathrm{~d} t^{2}} & =g-\frac{K_{f}}{M_{s}} \frac{I^{2}}{G^{2}}, \\
\frac{\mathrm{d} G}{\mathrm{~d} t} & =\frac{\mathrm{d} z_{t}}{\mathrm{~d} t}-\frac{\mathrm{d} Z}{\mathrm{~d} t}
\end{aligned}
$$

where $V_{c}$ is the coil's voltage, $F$ is the vertical force, $I$ is the coil's current, $G$ is the airgap, $Z$ is the electromagnet's position, and $B$ is the flux density. The fixed parameters of the model are as follows: $M_{s}$ is the vehicle's mass, $R_{c}$ is the coil's resistance, $N_{c}$ is the number of turns, $A_{p}$ is the pole face area and $z_{t}$ is the track's position. $K_{b}, K_{f}$ and $g$ reflect the flux, force and gravity constants (with values equal to $0.0015,0.0221$ and $9.81 \mathrm{~m} / \mathrm{s}^{2}$, respectively).

The linearization of the non-linear model is based on small perturbations around the operating point, eg, the airgap is assumed as $G=G_{o}+\left(z_{t}-z\right)$, where the lower case terms represent the small variation around the operating point, and subscript ' $O$ ' refers to the operating point. A similar approach is followed for $B, F, I, V_{c}$ and $Z$ ( $b, f, i, u_{c}$ and $z$ respectively).

The linearized state-space description of the EMS, with state $x \triangleq\left[\begin{array}{lll}i & \dot{z} & \left(z_{t}-z\right)\end{array}\right]^{\top}$ and the full sensor set of the maglev $y \triangleq\left[I, b,\left(z_{t}-z\right) \dot{z}, \ddot{z}\right]^{\top}$, is given by

$$
\begin{aligned}
\dot{x}(t) & =A x(t)+B_{u_{c}} u_{c}(t)+B_{\dot{z}_{t}} \dot{z}_{t}(t), \\
y(t) & =C x(t)
\end{aligned}
$$

where, $A$ is the $3 \times 3$ state matrix, $B_{u_{c}}$ is the $3 \times 1$ input matrix, is the $3 \times 1$ disturbance matrix, and $C$ is the $\alpha \times 3$ output matrix ( $\alpha$ varies from 1 to 5 (in this application), since its size changes according to the number of sensors in the sensor set). The aforementioned matrices are given by (11)-(13) in the Appendix. The various sensor sets can be obtained by appropriate selection of the corresponding rows in the output matrix $C$.

Note that the linearized model of the EMS is used for the design of the LQG controller, whereas for the tuning of the controller as well as for the validation via FIL, the non-linear model is applied in the loop. 


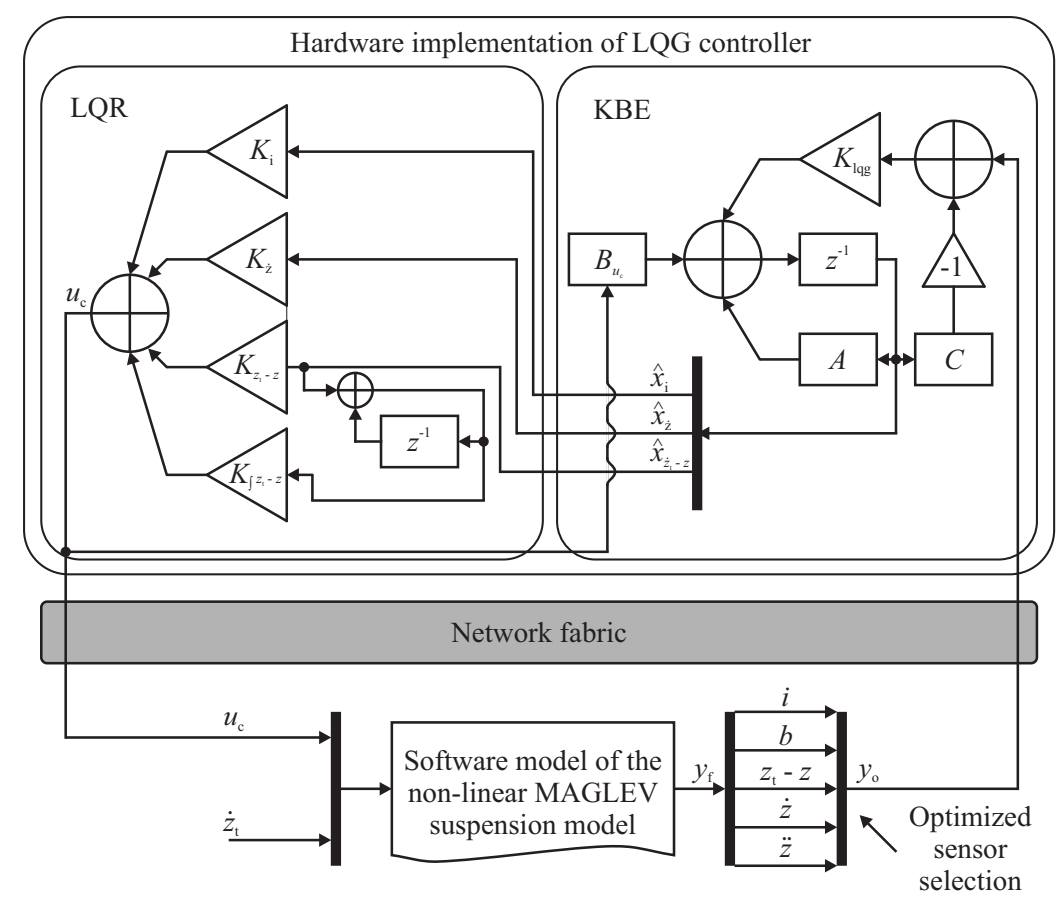

Fig. 2. Optimized sensor selection framework validation using FIL

\subsection{Closed-loop control requirements}

The closed-loop design requirements for the EMS depend on the type and operating velocity of the train [14], and are affected by the magnitude of the input disturbances to the suspension. There are two types of disturbances that enter in the vertical direction of the EMS:

(a) a stochastic behavior due to random variations of the rail position during vehicle movement;

(b) a deterministic behavior [4], considered in this work, occurs from the transition of the EMS onto the rail's gradients.

With the total weight taken as $1000 \mathrm{~kg}$, the operating point values for the EMS system are: $G_{o}=0.015 \mathrm{~m}, B_{o}=$ $1 \mathrm{~T}, I_{o}=10 \mathrm{~A}, V_{o}=100 \mathrm{~V}$ and $F_{o}=9810 \mathrm{~N}$. Hence, the parameters of the electromagnets, based on the operating point of the EMS, were calculated as follows: $R_{c}=10 \Omega$, $L_{c}=0.1 \mathrm{H}, N_{c}=2000$ and $A_{p}=0.01 \mathrm{~m}^{2}$. The deterministic disturbance here corresponds to a $5 \%$ track gradient at a vehicle speed of $15 \mathrm{~m} / \mathrm{s}$, an acceleration equal to $0.5 \mathrm{~m} / \mathrm{s}^{2}$, and a jerk (rate of change of acceleration) of $1\left(\mathrm{~m} / \mathrm{s}^{2}\right) / \mathrm{s}$. The EMS must support the payload and follow the track gradients. As a result, there are specific boundaries where the EMS is allowed to operate: (i) maximum airgap deviation, $\left(z_{t}-z\right)_{p} \leq 7.5 \mathrm{~mm}$; (ii) maximum control effort, $u_{c_{p}} \leq 300 \mathrm{~V}$; (iii) settling time, $t_{s} \leq 3 \mathrm{~s}$, and (iv) airgap steady state error, $e_{\left(z_{t}-z\right)_{s s}}=0$.

The control specifications are particularly addressed by the LQR controller in the first instance.
3 SYSTEMATIC FRAMEWORK FOR FIL

Any industrial plant has a number of control inputs $\left\{u_{i}: i=1, \ldots, n_{u}\right\}$, input disturbances $\left\{d_{i}: i=\right.$ $\left.1, \ldots, n_{d}\right\}$ and a set of possible outputs, $i e$, the full sensor set, $\mathcal{Y}_{f}=\left\{y_{i}: i=1, \ldots, n_{s}\right\}$. Part of the problem is to determine the set of sensors, $\mathcal{Y} \subset \mathcal{Y}_{f}$, for which the system is (i) stable, (ii) satisfies a number of closed-loop performance criteria and (iii) has a minimum number of sensing elements in the selected set, ie, the number of elements in $\mathcal{Y}_{o}$ is minimal ${ }^{1}$. The selection of $\mathcal{Y}_{o}$ with respect to the aforementioned properties is a very important and complex process, especially if the plant has a large number of actuator/sensor configuration possibilities, $i e$, sets.

This work is focused upon optimized sensor selection with respect to the aforementioned three properties. The full sensor set $\mathcal{Y}_{o}$ is a subset of the full sensor set $\mathcal{Y}_{f}$. Many subsets of the full sensor set are possible to be formed, and the number of them can be calculated from $N_{s}=2^{n_{s}}-1$, where $N_{s}$ is the total number of all sensor sets and $n_{s}$ is the total number of sensors.

The LQG controller combines a linear quadratic regulator (LQR), and a Kalman filter (KBE). The controller tuning is done via the separation principle [5]; the framework algorithm is executed in two stages:

\footnotetext{
${ }^{1}$ This paper deals only with minimizing the number of sensors, however there are other objectives that could be meaningful for applications other than the EMS systems. These can include minimizing energy consumption, size of weight and cost, etc.
} 
(i) In the first step, the LQR controller is optimized using a GA and the Pareto-optimality between the two objective functions, $\left(i e, \phi_{1}=i_{\mathrm{rms}}\right.$ and $\phi_{2}=\ddot{z}_{\mathrm{rms}}$ ) is found. The LQR controller state feedback gains (ie, $K_{\text {lqr }}=\left[K_{i}, K_{\dot{z}}, K_{\left(z_{t}-z\right)}, K_{\int\left(z_{t}-z\right)}\right]$, deduce the desired closed-loop response which is then selected and accounted as the desired or reference response for the next step.

(ii) The KBE is tuned for every feasible sensor set in order to achieve the desired closed-loop response.

Hence, a table is presented listing the optimised sensor sets with the selection of the "best" sensor set obtained by the overall control constraint violation function $\Omega$. The latter is given by,

$$
\Omega\left(k^{(l)}, f^{(j)}\right)=\sum_{l=1}^{n_{k}} \omega_{l}\left(k^{(l)}\right)+\sum_{j=1}^{n_{f}} \psi_{j}\left(f^{(j)}\right)
$$

where $\omega_{l}\left(k^{(l)}\right), \psi_{j}\left(f^{(j)}\right)$ are the $l^{\text {th }}, j^{\text {th }}$ soft and hard control constraint violations respectively, $n_{k}$ and $n_{f}$ are the number of soft and hard control constraints, and $\Omega$ is a function where any control constraint violation is reflected. If all control constraints are satisfied $\Omega$ becomes zero, otherwise its value depends on the level of the constraint(s) violation.

Figure 2 illustrates the FIL applied on the EMS where all five outputs, $\mathcal{Y}_{f}$, out of which only the $\mathcal{Y}_{o}$ (ie, the "best" sensor set) is fed into the FIL-based KBE.

\section{FPGA-BASED ARCHITECTURE OF THE LQG CONTROLLER}

The discrete linear time-invariant KBE has the following state space form,

$$
\begin{aligned}
\dot{\hat{x}}(k+1) & =A^{d} \hat{x}(k)+B_{u_{c}}^{d} u_{c}(k)+K_{l q g}^{d}\left(y(k)-C^{d} \hat{x}(k)\right), \\
\hat{y}(k) & =C^{d} \hat{x}(k)
\end{aligned}
$$

where $\hat{x}$ are the estimated states, $K_{l q g}^{d}$ is the $3 \times \beta$ observer gain matrix ( $\beta$ is the number of sensors) that minimizes $E\left\{[x-\hat{x}]^{\top}[x-\hat{x}]\right\}$ ( $x$ represents the actual states). $A^{d}, B_{u_{c}}^{d}$ are the state and input matrices given by (14) and (15) in the Appendix. $K_{l q g}^{d}$ has been calculated using the discrete time linear model of the EMS with a sampling rate of 10. Standard discretization procedure is followed here, hence full analysis is omitted.

The design architecture of the LQG core implementation and its entity in very high speed integrated circuits (VHSIC), hardware description language (VHDL) are depicted in Fig. 2 and Program 4 respectively. Figure 2 illustrates the internal architecture of $A^{3 \times 3}$ block, while a similar design approach is followed for $C^{\alpha \times 3}$ and $K_{l q g}^{3 \times \beta}$ blocks. In the present work, we used the MATLAB HDL coder tool to automate and speed up the process of translating the high level simulation model into an equivalent register transfer level (RTL) HDL description. Due to MATLAB HDL coder limitations in handling multi dimension matrices, a detailed LQG core model using

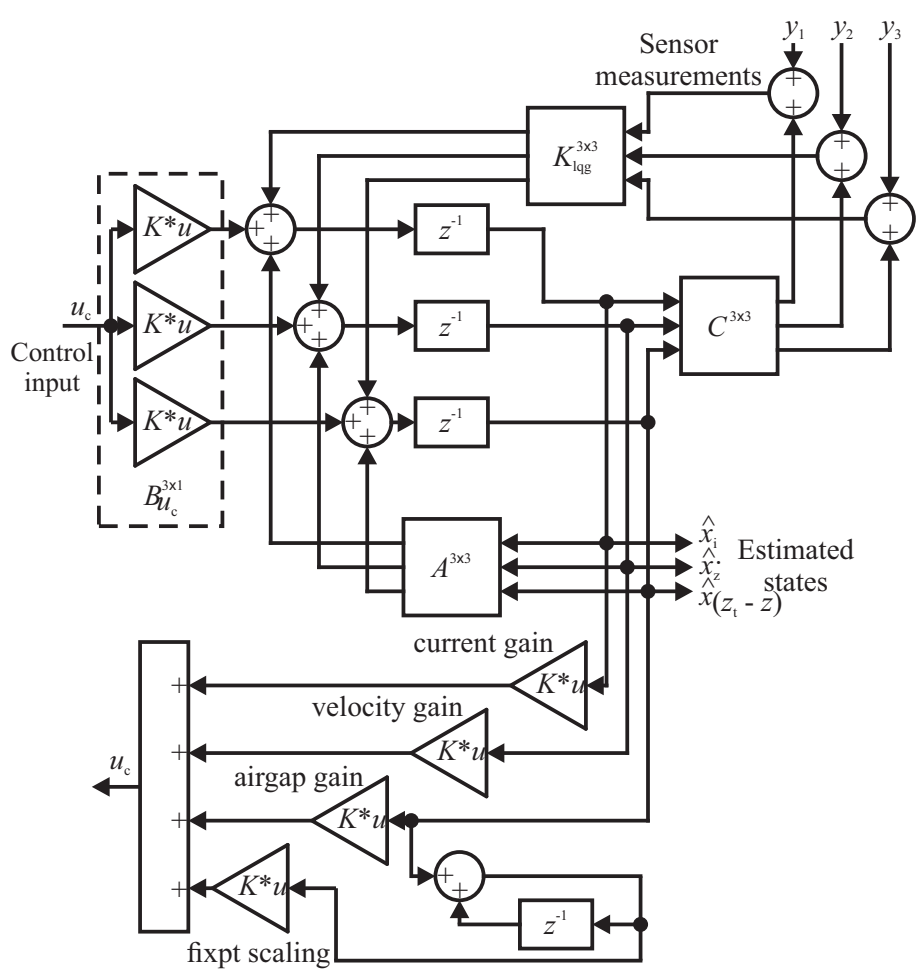

(a)

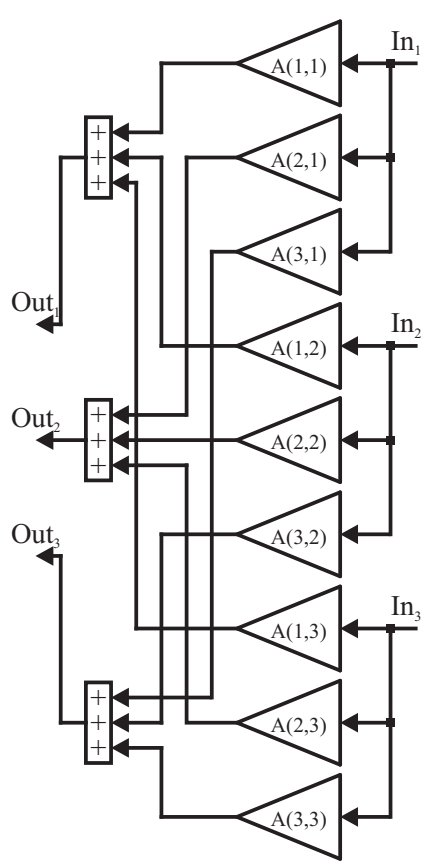

(b)

Fig. 3. (a) - LQG core architecture for 3 sensor measurements, iba (id:7) $\left(y_{1}, y_{2}, y_{3}\right)$, (b) - Internal architecture of $A^{3 \times 3}$ block 
explicitly scalar buses (see Fig. 2 and Program 1) was developed prior to HDL translation.

\subsection{Quantization process}

An algorithm in a high level system modelling environment (such as MATLAB/Simulink) is represented in the floating-point domain, where mostly all variables are 64-bit allowing all operations to be performed in high precision format with large accuracy. In a digital VLSI implementation this translates to an increased number of flip-flops and combinational logic and inevitably results on a design that requires a large silicon area on the FPGA chip, large critical path that negatively affects speed, plus increased power consumption.

To address the aforementioned problem, the algorithm under implementation (LQG in this work) has first undergone a conversion to the fixed-point domain and then modelled using VHDL. In the fixed-point domain, a pair of wordlength, WL, and quality fractional range, QF, is considered for each parameter of the algorithm. As a consequence as (WL,QF) is increased will give a smaller Bit-Error Rate (BER) but larger silicon area, whereas as (WL,QF) is reduced will result to increased BER and smaller silicon area. Several simulations need to run to decide on the number of bits for (WL,QF) and the dynamic range of the parameters (MATLAB fixedpoint tool), in order to maintain a desired precision which will not compromise the overall system performance ( $i e$, destabilize the control loop), and maintain a low silicon area.
The fixed-point range for a signed number $\pm a$ in a 2 's complement form is defined by the minimum and maximum value range a signed integer number type of Quantity Integer range (QI) bits can hold. The latter is best expressed by the inequality, $-2^{Q I-1} \leq a \leq 2^{Q I-1}-1$ and can be rewritten,

$$
-2^{Q I-1} \leq a<2^{Q I-1}, \quad a \in \mathbb{Z} .
$$

From (5) it can be easily shown that,

$$
\begin{aligned}
& \left.Q I\right|_{a_{\min }} \geq \log _{2}(-a)+1 \text { if } a<0, \\
& \left.Q I\right|_{a_{\max }}>\log _{2}(a)+1 \quad \text { if } a>0 .
\end{aligned}
$$

Since the positive constraint is the tighter one due to the asymmetry of signed integer types about zero, the constraint for the required number of bits can be generalised as follows,

$$
Q I>\log _{2}\left(\max \left[\left|a_{\min }\right|,\left|a_{\max }\right|\right]\right)+1
$$

Since $Q I$ is an integer number of bits we can truncate the result and add one to form an equation to compute $Q I$ (that satisfies the constraint $Q I>\log _{2}|a|$ ) such as,

$$
Q I=\left\lfloor\log _{2}\left(\max \left[\left|a_{\min }\right|,\left|a_{\max }\right|\right]\right)+2\right\rfloor .
$$

\footnotetext{
${ }^{2}$ Each slice contains 4 Look Up Tables (LUTs) and 8 Flip-Flops (FFs).
}

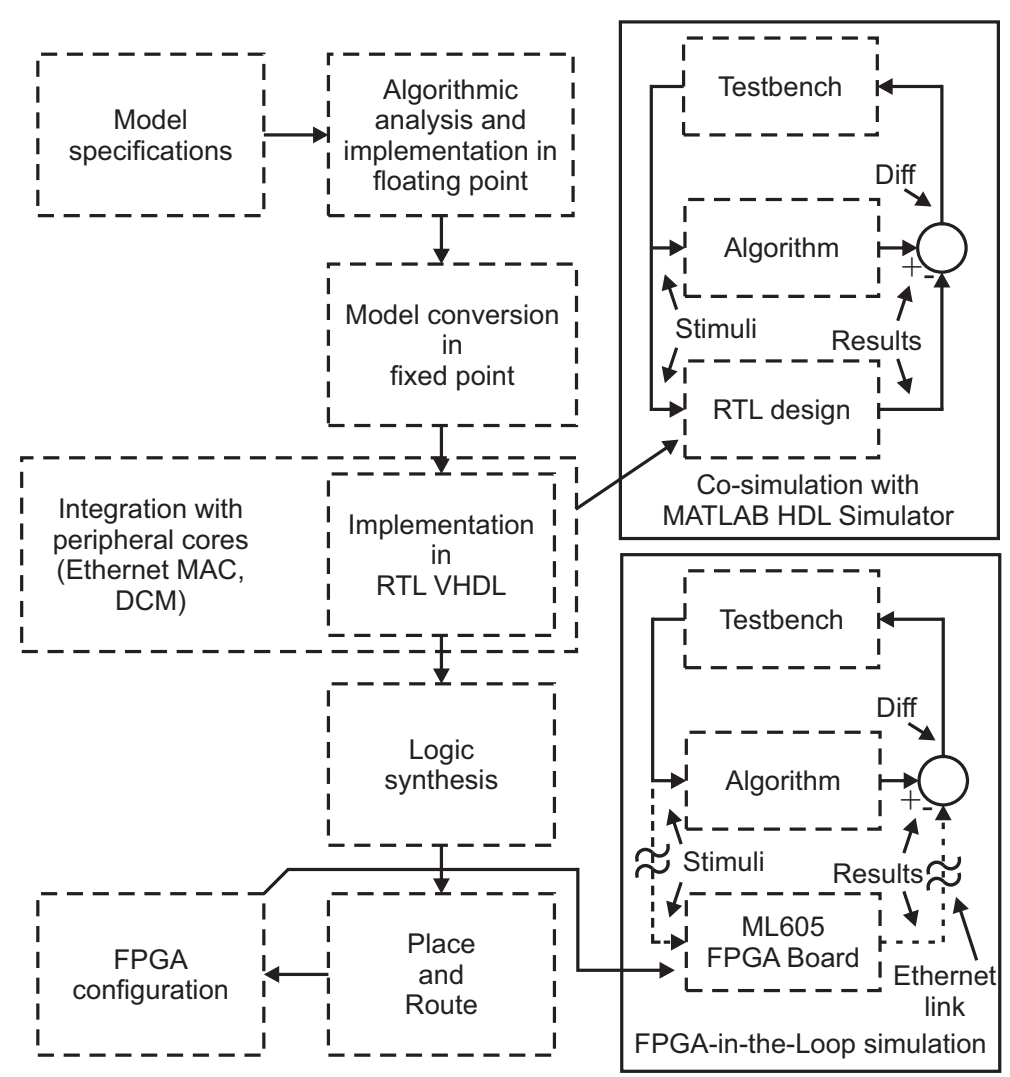

Fig. 4. FPGA hardware/software co-design and implementation flow 
Assuming a resolution $\varepsilon=2^{-Q F}$ for the fractional part, the qauantity fractional $(Q F)$ range becomes, $Q F=$ $\left\lceil\log \left(\varepsilon^{-1}\right)\right\rceil$; hence, the required wordlength $W L$, to sufficiently represent a float number to a fixed point representation, is given by the sum of $Q I$ and $Q F$ such that,

$$
\left.W L\right|_{\operatorname{Req}} \geq Q I+Q F
$$

or

$$
-2^{Q I-1} \leq a<2^{Q I-1}-\left.2^{-Q F}\right|_{\varepsilon=2^{-Q F}} .
$$

\subsection{FPGA Implementation}

The FIL presented in this work was implemented on a Xilinx Virtex-6 ML605 development board. The ML605 board utilizes a Xilinx Virtex-6 device (XC6VLX240T1FFG1156) [15] in the 1156-pin fine-pitch Ball Grid Array (BGA) package, featuring slices ${ }^{2}$ and special Digital Signal Processing (DSP) slices (DSP48A1). The LQG and the peripheral cores were synthesized using Xilinx Synthesis Tool (XST).

A top-down manner [16] has been followed for the design process of the LQG controller (Fig. 4). The process initiates with the model specifications and requirements, advances to a high level functional system model (Simulink model) and continues on converting it to fixedpoint prior to FPGA implementation. Co-simulation of the RTL model side-by-side with the fixed-point Simulink model was performed using MATLAB's HDL verifier and Mentor's Modelsim simulator in [17]. Moreover the implemented system on the FPGA chip was compared in real time using a cycle accurate Simulink model forming a FIL setup.

\section{RESULTS ANALYSIS}

In this section, the results from the FIL used to validate the sensor selection framework are analyzed. As explained in Section 3, the first step in the output selection process is to design the state vector $K_{l q r}$. This is based on three performance criteria: (i) closed-loop vertical acceleration, $\ddot{z}_{r m s}<0.5 \mathrm{~m} / \mathrm{s}^{2}$, (ii) excitation coil's current, $i_{r m s}<2 \mathrm{~A}$ and (iii) best possible ride quality, $i e, \min \left(\ddot{z}_{r m s}\right)$.

Note that the analysis related to Figs. 5, 6, 7, 8, is performed on the continuous-time [5] system to emphasize the design expectation aspects prior to implementation. This is not problematic as a sampling rate - for discretisation - of $10 \mathrm{kHz}$ is used, which is well above the typical maglev loop bandwidth of $<20 \mathrm{~Hz}$. The discrete time LQG controller is listed in the Appendix.

\subsection{LQR Controller}

Although the emphasis of the paper is the HIL embedded nature of the design, here we present some useful details on the design of the controller. In particular, the LQR controller is designed via the usual procedure of minimising the cost function $J_{l q r}=\int\left[x^{\top} Q x+u^{\top} R u\right]$ with the tuning matrices setup via Bryson's rule [3]. We select LQR gains on the "best" (lowest value) ride quality level using nominal parameters for the model. Then the LQR design is assessed in terms of the robustness level by allowing $\pm 10 \%$ dynamic system parameter variation from nominal values for the following parameters: mass of vehicle (force follows trend), resistance and inductance, initial condition of current, initial condition of flux. The total combinations given the parameters is $=1125$. Figure 5 illustrates the characteristic loci of the return ratio and CL poles. It is clearly shown that the ideal robust properties of LQR are not violated.

Figure 6 illustrates the magnitude spectrum of the closed-loop: vertical acceleration and air-gap, respectively, to $\dot{z}_{t}$. The typical ride quality criterion of $<5 \% \mathrm{~g}$ RMS acceleration is not violated (note that deterministic responses are discussed later in this section).

\subsection{Kalman filter (KBE) in the closed loop}

The KBE in the second step of the framework is used to estimate the states, thus an optimized tuning of the $\mathrm{KBE}$ is necessary to accurately estimate the states and feed the LQR controller.

\begin{tabular}{|c|c|c|c|}
\hline id & $\begin{array}{l}\text { Sensor } \\
\text { Set }\end{array}$ & $\begin{array}{l}\text { Deterministic } \\
\text { response }\end{array}$ & $\Omega$ \\
\hline & LQR response $\rightarrow$ & $\checkmark$ & $\checkmark$ \\
\hline 1 & $b$ & $\checkmark$ & $\checkmark$ \\
\hline 2 & $\left(z_{t}-z\right)$ & $x$ & $x$ \\
\hline 3 & $\ddot{z}$ & $\checkmark$ & $\checkmark$ \\
\hline 4 & $i, b$ & $\checkmark$ & $\checkmark$ \\
\hline 5 & $i, \ddot{z}$ & $\checkmark$ & $\checkmark$ \\
\hline 6 & $i, b,\left(z_{t}-z\right)$ & $\checkmark$ & $\checkmark$ \\
\hline 7 & $i, b, \ddot{z}$ & $\checkmark$ & $\checkmark$ \\
\hline 8 & $i, b, \dot{z}, \ddot{z}$ & $\checkmark$ & $\checkmark$ \\
\hline 9 & $i, b,\left(z_{t}-z\right), \dot{z}, \ddot{z}$ & $\checkmark$ & $\checkmark$ \\
\hline
\end{tabular}

Table 1. Optimized sensor selection simulation results

The optimization process shown that 24 out of 31 sensor sets provided the same closed-loop response (including the dynamic controller) compared to the closed-loop with the ideal LQR (the design was on the nominal system). Some of the corresponding results from the offline framework are presented in Table 1. The first column is the sensor set identification number (id), the second column is the corresponding sensor set, and the next two columns show whether the deterministic response of the EMS is satisfied $(\checkmark)$ or not $(\times)$. The $\Omega$ function in the last column similarly indicates whether all control constraints described in Section 2.2 are fulfilled $(\checkmark)$ or not $(\times)$. The selection of the best sensor set is done based on $\Omega$ as described in Section 3. From a close inspection on the table, one can easily identify the best sensor set selection $i e$, the one with the minimum number of sensor/s which satisfies all control requirements is id:1. In this work, the 

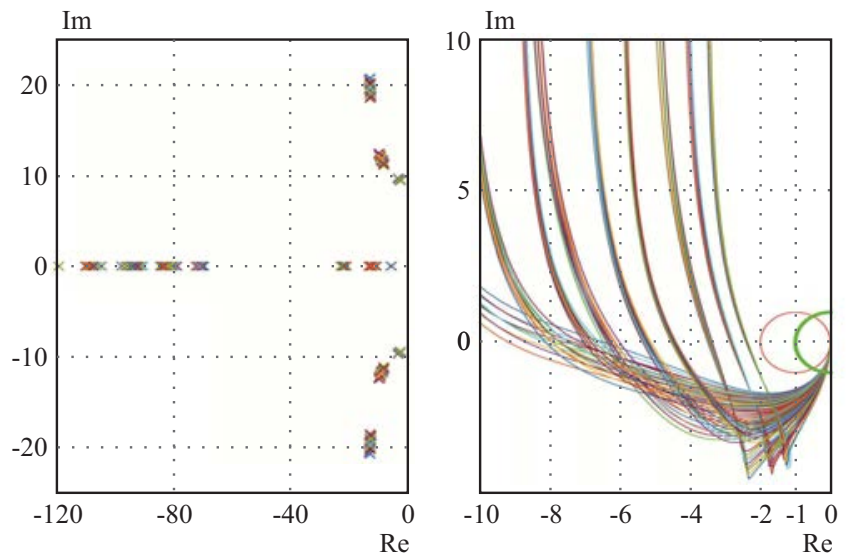

Fig. 5. LQR Robustness Return ratio and closed-loop poles,(tuning performed on nominal system "best" ride quality) / continuous-time
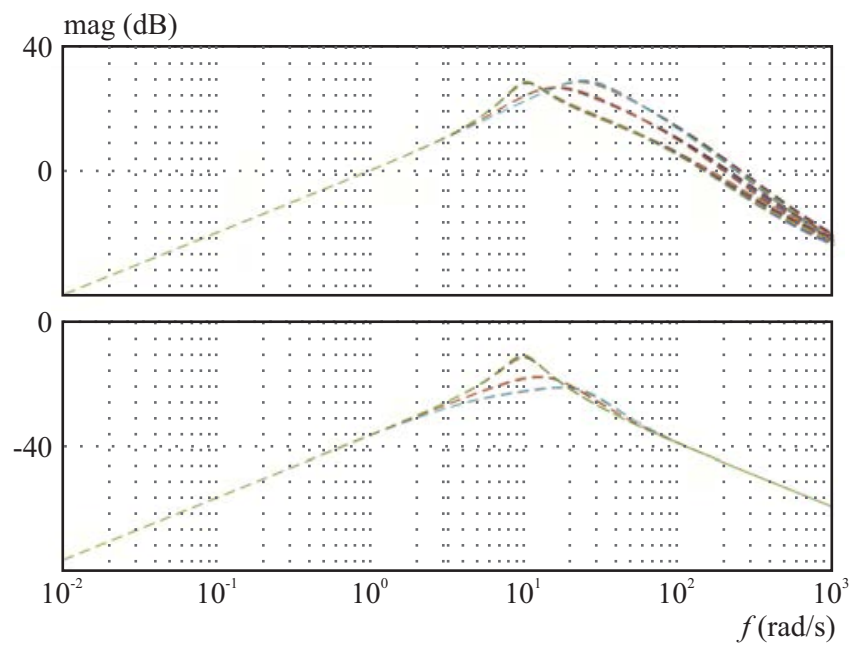

Fig. 6. CL responses with LQR only $\left(\dot{z}_{t} \rightarrow \ddot{z}\right.$ (top) and $\dot{z} \rightarrow$ $\left(z-z_{t}\right)$ (bottom)) under model variation

Table 2. Design utilization summary: three sensors, iba (id:7); and one sensor, b (id:1)

\begin{tabular}{lccccc}
\hline \multirow{2}{*}{ Logic utilization } & \multicolumn{3}{c}{ Used } & \multirow{2}{*}{ Available } & \multicolumn{2}{c}{ Utilization } \\
& $i b a($ id:7) & $b(\mathrm{id}: 1)$ \\
\hline Slice Register & 2410 & 2,354 & 301,440 & $1 \%$ & $1 \%$ \\
Slice LUTs & 4012 & 3777 & 150,720 & $2 \%$ & $2 \%$ \\
Occupied Slices & 1402 & 1362 & 37,680 & $3 \%$ & $3 \%$ \\
Bonded IOBs & 30 & 30 & 600 & $5 \%$ & $5 \%$ \\
Block RAMB36E1 & 2 & 2 & 416 & $1 \%$ & $1 \%$ \\
Block RAMB18E1 & 1 & 1 & 832 & $1 \%$ & $1 \%$ \\
BUFG & 5 & 4 & 32 & $15 \%$ & $12 \%$ \\
DSP48E1s & 73 & 69 & 768 & $9 \%$ & $8 \%$ \\
MMCM_ADVs & 1 & 1 & 12 & $8 \%$ & $8 \%$ \\
\hline
\end{tabular}

FIL was implemented for id:1 (single sensor), and is compared with id:7 (three sensors). The LQG-type controller, $i e$, incorporating the Kalman filter and the LQR gains, implementation flow on the FPGA for other sensor set combinations follows the same approach.

Here we considered the design of the Kalman filter so as to provide the "best" estimates to the LQR controller. Some information on the impact of introducing the Kalman filter -in the closed-loop - on preliminary robustness performance (given the parameter variation considered from the LQR design section) is also presented. As in the case of LQR only, we look into the stochastic ride quality criterion. Figure 7 shows the (continuous-time) magnitude spectrum of the closed-loop: vertical acceleration and air-gap, respectively, to $\dot{z}_{t}$. Note that the KF considered in this set of figures is based on id:7 sensor set.

Figure 8 shows the results on stochastic ride quality under parameter variation (CL with $\mathrm{KF}+\mathrm{LQR}$ ). The typical criterion of $<5 \% \mathrm{~g}$ acceleration is not violated, with $90 \%$ of the samples being less than $3.9 \% \mathrm{~g}$ acceleration level.

\subsection{FPGA implementation for 2 sensor sets}

The resources requirements and trial and error quantization procedure are commented in this section. Table 2 show the logic utilization for the implemented integrated system on the FPGA device, for three and one sensor.

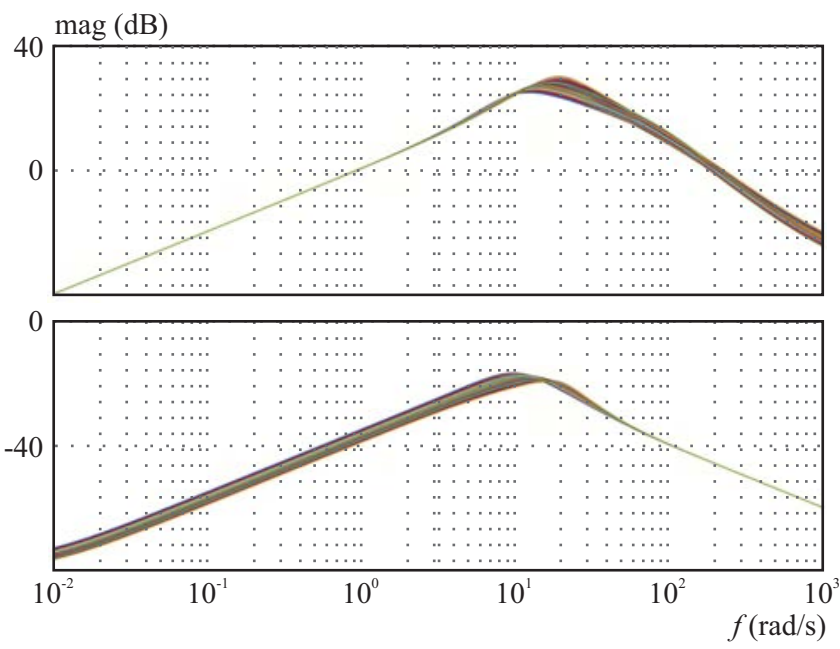

Fig. 7. CL responses with LQR + Kalman filter $\left(\dot{z}_{t} \rightarrow \ddot{z}\right.$ (top) and $\dot{z}_{t} \rightarrow\left(z-z_{t}\right)$ (bottom)) under model variation

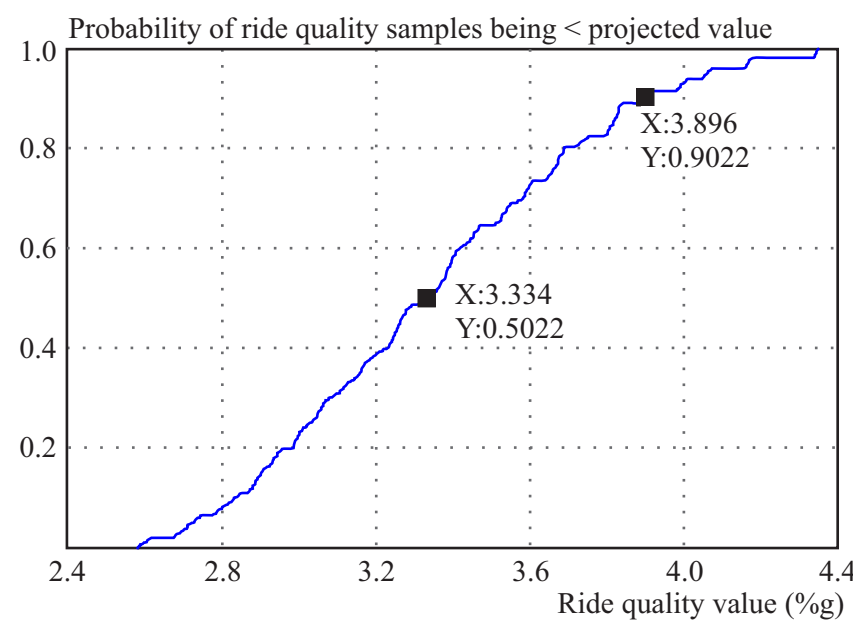

Fig. 8. Stochastic ride quality of $\mathrm{CL}$ with $\mathrm{KF}+\mathrm{LQR}$ under parameter variation - Cumulative distribution function plot (acceleration RMS values) 

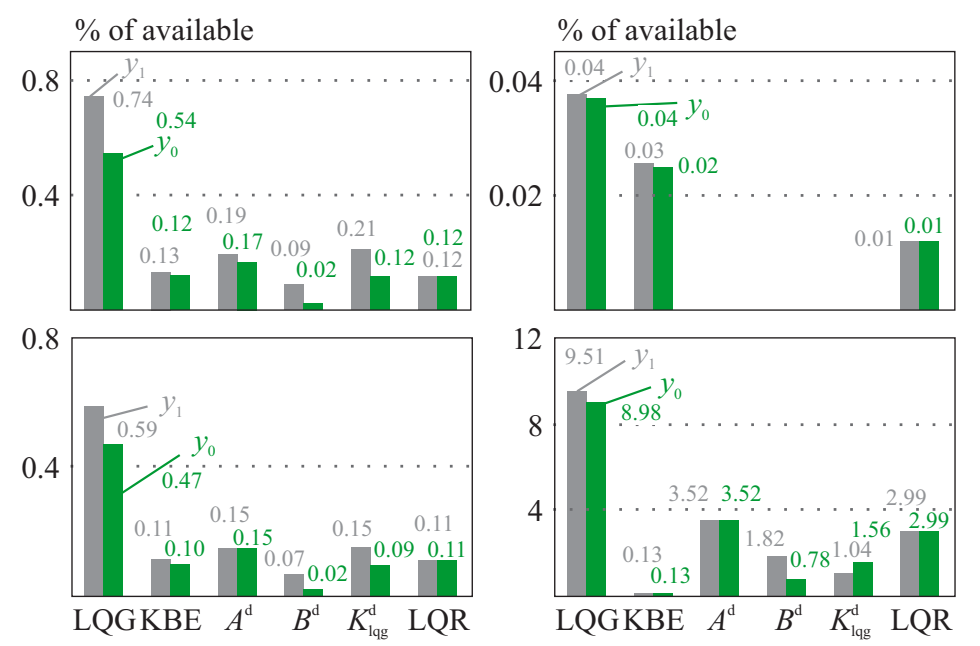

Fig. 9. FPGA resources comparison for id:7 and id:1
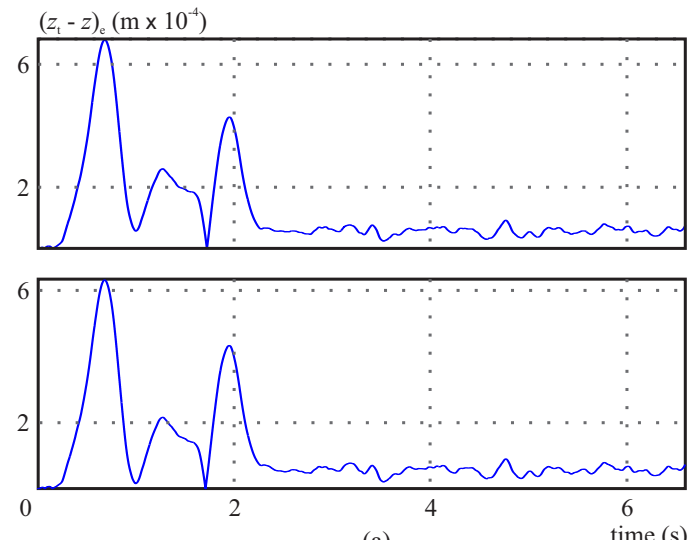

(a)
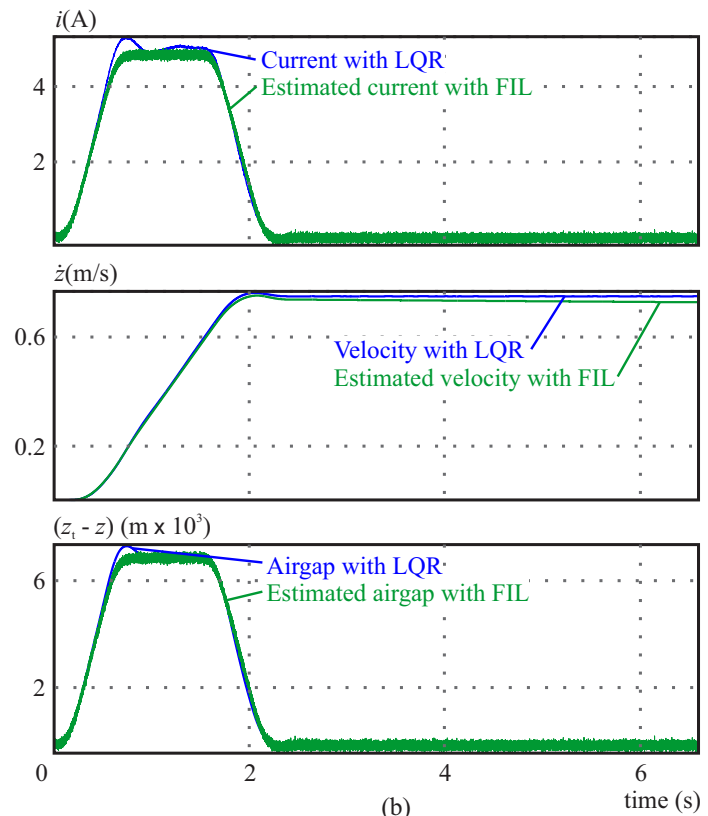

Fig. 10. (a) — Airgap error with sets (id:1), (id:7) (b) — State estimation with set (id:1)

The implemented design mainly includes the LQG core, ethernet medium access control (MAC) [18] and the clock manager modules. The ethernet MAC core is licensed as part of the Xilinx embedded development kit
(EDK). According to the device utilization report from the Xilinx map (MAP) tool (see Table 3), the LQG core module itself for id:7, occupies 265 slices, 113 slice registers, 884 slice LUTs, and 73 DSP cores (DSP48A1) and similarly for id:1, 205 slices, 111 slice registers, 696 slice LUTs, and 69 DSP48A1. A comparison of the utilized FPGA resources between three sensors, $i b a$ (set id:7) and one sensor, $b$ (set id:1) LQG implementation scenarios, is depicted in Fig. 9. It is easy to see that the overall occupied area for set id:1 onto the FPGA chip is significantly smaller when compared to what is required by set id:7. The implemented design uses one mixed mode clock manager (MMCM) module [19] that produces the different clocks inside the FPGA chip. The placed and routed FPGA designs (LQG and peripheral cores) for three and one sensor implementations, achieve according to postplace and route timing report a system clock operating frequency of $34.364 \mathrm{~ns}$ or $29.1 \mathrm{MHz}$, and $32.819 \mathrm{~ns}$ or $30.5 \mathrm{MHz}$ respectively. The aforementioned speeds were obtained with the map and place and route effort set to medium on Xilinx ISE 13.3.

\subsection{Data analysis from FIL simulations}

Figure 10 depicts the performance of the EMS using two sensor sets. One comprising a single sensor (set id:1) and the other one having three sensors (set: id:7). In particular, Fig. 10a compares the airgap deflection error from simulation-based continuous-time and FIL-based discrete-time KBE with set id:1 (top) and set id:7 (bottom). The performance of the EMS is within the required performance objectives given in Section 2.2. The airgap error magnitude between the simulation and FIL is in the order of d-4 which is fairly small. The state estimation of the LQG using set id:1 is shown in Fig. 10b. All three states $\left(i, \dot{z}\right.$ and $\left.z_{t}-z\right)$ are accurately estimated using one sensor $(b)$, which has similar performance to the state estimation achieved with more sensors added, $i e$, set id:7. Hence, the EMS system can perform adequately, with respect to the given requirement, with fewer sensors. 


\section{CONCLUSIONS}

A semi-practical modeling and validation of an embedded control system for sensor optimization on a EMS plant using the FIL concept was presented. A high-level MATLAB/Simulink environment used to model the physical process and the LQG controller implemented on an FPGA. A model-based design approach has been followed for the FIL implementation using a fusion of system modeling and hardware/software co-design. Robustness issues on the LQR and designed LQG (i.e., Kalman-Filter and LQR) as well as the effect of fixed-point quantization was analyzed early in the design process. In the quantization case the wordlength was optimized to yield a smaller implementation. System level test benches were used with HDL co-simulation to verify the HDL implementation, and also FIL simulation to significantly accelerate the system validation. Without loss of generality, we compared FIL results for the LQG implementation of two sensor sets (id:1 and id:7). It was shown that the LQG implementation in FIL is successful in spite of the complex quantization procedure.In addition it was shown that the FIL approach greatly reduces simulation time when compared to the software-based model counterpart. Current work studies design architecture of LQG with dynamic switching of the gains given the sensor set provision.

Table 3. Design utilization summary: three sensors, iba (id:7), and one sensor, $b$ (id:1)

(a) id:7

\begin{tabular}{|c|c|c|c|c|}
\hline Module $(i b a)$ & Slices & Slice reg. & LUTs & DSP48E1 \\
\hline LQG & $0 / 265$ & $0 / 113$ & $0 / 884$ & $0 / 73$ \\
\hline $\mathrm{KBE}$ & $49 / 221$ & $77 / 77$ & $170 / 717$ & $1 / 50$ \\
\hline$A^{d}$ & $67 / 67$ & $0 / 0$ & $221 / 221$ & $27 / 27$ \\
\hline$C^{d}$ & $30 / 30$ & $0 / 0$ & $99 / 99$ & $14 / 14$ \\
\hline$K_{l q g}^{d}$ & $75 / 75$ & $0 / 0$ & $227 / 227$ & $8 / 8$ \\
\hline LQR & $44 / 44$ & $36 / 36$ & $167 / 167$ & $23 / 23$ \\
\hline \multicolumn{5}{|c|}{ (b) id:1 } \\
\hline Module $(b)$ & Slices & Slice reg. & LUTs & DSP48E1 \\
\hline LQG & $0 / 205$ & $0 / 111$ & $0 / 696$ & $0 / 69$ \\
\hline $\mathrm{KBE}$ & $41 / 161$ & $75 / 75$ & $143 / 529$ & $1 / 46$ \\
\hline$A^{d}$ & $69 / 69$ & $0 / 0$ & $220 / 220$ & $27 / 27$ \\
\hline$C^{d}$ & $12 / 12$ & $0 / 0$ & $30 / 30$ & $6 / 6$ \\
\hline$K_{l q g}^{d}$ & $39 / 39$ & $0 / 0$ & $136 / 136$ & $12 / 12$ \\
\hline LQR & $44 / 44$ & $36 / 36$ & $167 / 167$ & $23 / 23$ \\
\hline
\end{tabular}

Note . This paper is an extended version of earlier work presented by the authors in [20]. In particular, the authors extend their previous work by in-depth investigation of robustness, both in the design stage and expectations as well as in the robustness of the quantization (which works appropriately in both "worst" and "best" ride quality case choices).

\section{Appendix A. EMS State space matrices (sym- bolic parameters)}

$$
\begin{aligned}
& A=\left[\begin{array}{ccc}
-\frac{R_{c}}{L_{c}+\frac{K_{b} N_{c} A_{p}}{G_{o}}}-\frac{K_{b} N_{c} A_{p} I_{o}}{G_{o}^{2}\left(L_{c}+\frac{K_{b} N_{c} A_{p}}{G_{o}}\right)} & 0 \\
-2 K_{f} \frac{I_{o}}{M_{s} G_{o}^{2}} & 0 & 2 K_{f} \frac{I_{o}^{2}}{M_{s} G_{o}^{3}} \\
0 & -1 & 0
\end{array}\right] \\
& B_{u_{c}}=\left[\begin{array}{lll}
\frac{1}{L_{c}+\frac{K_{b} N_{c} A_{p}}{G_{o}}} & 0 & 0
\end{array}\right]^{\top}, \\
& B_{\dot{z}_{t}}=\left[\begin{array}{lll}
\frac{K_{b} N_{c} A_{p} I_{o}}{G_{o}^{2}\left(L_{c}+\frac{K_{b} N_{c} A_{p}}{G_{o}}\right)} & 0 & 1
\end{array}\right]^{\top} .
\end{aligned}
$$

While the output matrix $C$, for the different output measurements as mentioned in the main body of the paper, is formed by the relevant raw combination(s) from the state and input matrices accordingly.

\section{Appendix B. LQG controller state-space ma- trices numerical results (relating to sensor sets, id:1 and id:7)}

$$
\begin{gathered}
A^{d}=\left[\begin{array}{ccc}
9.99 \times 10^{-1} & -6.34 \times 10^{-2} & 4.15 \times 10^{-3} \\
1.96 \times 10^{-4} & 1.00 & -1.30 \times 10^{-1} \\
-9.80 \times 10^{-9}-1.00 \times 10^{-4} & 1.00
\end{array}\right], \\
B_{u_{c}}^{d}=\left[\begin{array}{lll}
4.76 \times 10^{-5} & 4.67 \times 10^{-9} & -1.55 \times 10^{-13}
\end{array}\right]^{\top}, \\
B_{\dot{z}_{t}}^{d}=\left[\begin{array}{lll}
6.34 \times 10^{-2} & -3.12 \times 10^{-7} & 1.00
\end{array}\right]^{\top} \\
K_{l q g}^{d}=\left[\begin{array}{ccc}
3.56 \times 10^{-1} & -57.0 & -2.82 \\
-3.11 \times 10^{-6} & 1.49 \times 10^{-3} & 7.40 \times 10^{-5} \\
5.61 \times 10^{-4} & -9.00 \times 10^{-2}-4.45 \times 10^{-3}
\end{array}\right], \\
C^{d}=\left[\begin{array}{ccc}
1.00 & 0 & 0 \\
0.10 & 0 & -66.6 \\
1.96 & 0 & -1.30 \times 10^{3}
\end{array}\right], \\
K_{l q g}^{d}=\left[\begin{array}{ccc}
-1.61 \times 10^{2} 3.61 \times 10^{-3} & -2.54 \times 10^{-1}
\end{array}\right]^{\top}, \\
C^{d}=\left[\begin{array}{lll}
0.10 & 0 & -66.6
\end{array}\right]^{\top} .
\end{gathered}
$$

\section{REFERENCES}

[1] ISERMANN, R.-SCHAFFNIT, J.-SINSEL, S. : Hardware-inthe-Loop Simulation for the Design and Testing of Engine-Control Systems, Control Eng. Pract. 7 No. 5 (1999), 643-653.

[2] LU, B.-WU, X.-FigueroA, H.-MONTI, A. : A Low-Cost Real-Time Hardware-in-the-Loop Testing Approach of Power Electronics Controls, IEEE Trans. Ind. Electron. 54 No. 2 (2007), 919-931.

[3] Michail, K.-ZOlotas, A.-GOODAll, R. M.: Optimised Configuration of Sensors for Fault Tolerant Control of an Electromagnetic Suspension System, Int. J. Syst. Sci. 43 No. 10 (2012), 1785-1804.

[4] MICHAIL, K.: Optimised Configuration of Sensing Elements for Control and Fault Tolerance Applied to an Electro-Magnetic Suspension System, 2009.

[5] SKOGeStad, S.-POstlethwhite, I. : Multivariable Feedback Control Analysis and Design, $2^{\text {nd }}$ edition, John Wiley \& Sons Ltd, New York, 2005. 
[6] KONAK, A.-COIT, D. W.-SMITH, A. E.: Multi-Objective Optimization using Genetic Algorithms: A Tutorial, Reliab. Eng Syst. Saf. 91 No. 9 (2006), 992-1007.

[7] ZHANG, Y.-JIANG, J.: Bibliographical Review on Reconfigurable Fault-Tolerant Control Systems, Annu. Rev. Control 32 No. 2 (2008), 229-252.

[8] BOUKHNIFER, M.: Active Fault Tolerant Control for Ultrasonic Piezoelectric Motor, J. Electr. Eng. 63 No. 4 (2012), 224-232.

[9] FORRAI, A.: Embedded Control System Design: A Model Based Approach, Springer Sci. \& Business Media, 2012.

[10] ALUR, R.-HRISTU-VARSAKELIS, D.-ARZEN, K.-E.-LEVINE, W.-BAILLIEUL, J.-HENZINGER, T. A. Eds. : Handbook of Networked and Embedded Control Systems, Corr., $2^{\text {nd }}$ printing, Birkhauser, Boston, 2008.

[11] BACIC, M.: On Hardware-in-the-Loop Simulation, $4^{\text {th }}$ IEEE Conference on Decision and Control and European Control Conference, 2005, pp. 3194-3198.

[12] LUCIA, O.-JIMENEZ, O.-BARRAGAN, L. A.-URRIZA, I.-BURDIO, J. M.-NAVARRO, D. : Real-Time FPGA-based Hardware-in-the-Loop Development Test-Bench for Multiple Output Power Converters, in $6^{\text {th }}$ Annual IEEE Applied Power Electronics Conference and Exposition (APEC), 2010, 309-314.

[13] DELIPARASCHOS, K. M.-MICHAIL, K.-TZAFESTAS, S. G.-ZOLOTAS, A. C.: Optimised Sensor Selection for Control: A Hardware-in-the-Loop Realization on FPGA for an EMS System, in Conference on Control and Fault-Tolerant Systems (SysTol), 2013, pp. 727-732.

[14] GOODALL, R. M.: Dynamics and Control Requirements for EMS Maglev Suspensions, in Proceedings on International Conference on Maglev, 2004, pp. 926-934.

[15] Xilinx Inc., Virtex-6 Family Overview. 2012.

[16] DELIPARASCHOS, K. M.-NENEDAKIS, F. I.-TZAFESTAS, S. G. : Design and Implementation of a Fast Digital Fuzzy Logic Controller using FPGA Technology, J. Intell. Robot. Syst. 45 No. 1 (Jan 2006), 77-96.

[17] MOUSTRIS, G. P.-DELIPARASCHOS, K. M.-TZAFESTAS, S. G.: Feedback Equivalence and Control of Mobile Robots through a Scalabe FPGA Architecture, in Recent Advances in Mobile Robotics (Dr. Andon Venelinov Topalov, ed.), InTech, 2011.

[18] Xilinx Inc., LogiCORE IP Virtex-6 FPGA Embedded Tri-Mode Ethernet MAC Wrapper v2.3. 2012.

[19] Xilinx Inc., Virtex-6 FPGA Clocking Resources User Guide. 2014.

[20] DELIPARASCHOS, K. M.-MICHAIL, K.-TZAFESTAS, S. G.-ZOLOTAS, A. C.: A Model-Based Embedded Control Hardware/Software Co-Design Approach for Optimized Sensor Selection of Industrial Systems, in $23^{\text {th }}$ Mediterranean Conference on Control and Automation (MED), Toremolinos, Spain, 2015, pp. 889-894.

\section{Received 17 January 2016}

Kyriakos M. Deliparaschos born in Athens, Greece, received his BEng Hons degree in Electronics Engineering from De Montfort University, Leicester, UK, the MSc degree in Mechatronics from De Montfort University and National Technical University of Athens (NTUA), and his PhD degree from the Department of Signals, Control and Robotics, School of Electrical and Computer Engineering, NTUA. Currently he is Adjunct Lecturer at the Department of Electrical and Computer Engineering and Informatics of Cyprus University of Technology (CUT), a Research Associate with the Robotics Control and Decision Systems (RCDS) Lab part of the Mechanical Engineering and Material Science and Engineering
Department of the CUT, and also a Research Associate at the Intelligent Robotics and Automation Lab of NTUA. Previously he was a Postdoctoral Research Fellow (DISCUS FP7 funded project,) with CTVR group at Trinity College, Dublin University.

Konstantinos Michail was born in Nicosia, Cyprus. He obtained his BSc in Electronic Engineering from Budapest University of Technology and Economics, Hungary, 2003. In 2004 he received an MSc from Newcastle University Upon Tyne, UK and in 2009 he was awarded the $\mathrm{PhD}$ degree from Loughborough University, UK both in automatic control systems. His research activities fall in the areas of modern and classical control methods, robust control, fault tolerant control, multi-objective optimization, renewable energy systems, communication systems, robotics and digital signal processing. He has published a number of journal and conferences papers in control systems. Since 2013 he is a senior research engineer with SignalGeneriX Ltd working on communications and control systems R\&D.

Argyrios C. Zolotas, born in Xanthi, Greece, graduated from the University of Leeds in 1998 and received his $\mathrm{PhD}$ degree in 2002 from Loughborough University. He also obtained an MSc (Distinction) from University of Leicester via a part-time program under the lifelong learning initiative of the LLP-CPD framework. Since September 2014 he is Reader (and from January 2015 also deputy director of research) in the School of Engineering, University of Lincoln UK. He previously held academic positions at University of Sussex and Loughborough University, and a post-doctoral research fellowship at Imperial College London. He is senior member of the IEEE and a fellow of the UK Higher Education Academy. His research interests are in the areas of robust control of linear and nonlinear systems, system structure selection, system resilience, applications of advanced control, emerging control applications.

Spyros G. Tzafestas was born in Corfu, Greece. He holds $\mathrm{PhD}$ and $\mathrm{DSc}$ degrees in Control and Automation and he is professor emeritus, Director of the Institute of Communication and Computer Systems (ICCS), senior research associate of the Signals, Control and Robotics Division and the Intelligent Robotics and Automation Laboratory (IRAL) of the National Technical University of Athens (NTUA). He is recipient of honorary doctorates from the International University (DSc (Hon)), the Technical University of Munich (Dr-Ing Eh) and the Ecole Centralede Lille (Docteur Honoris Causa). Fellow of IEEE (N.Y.) and IEE (London); Member of ASME (N.Y.), New York Academy of Sciences, IMACS (Rutgers, N.J.) and SIRES (Brussels). Member of IFAC SECOM and MIM TCs. Project evaluator of national European and international projects (USA, Canada, Italy, Hong Kong, Japan). Project coordinator of national and EU projects in the fields of robotics, CIM and IT (ESPRIT, BRITE-EURAM, TIDE, INTAS, SOCRATES, EUREKA, GROWTH, etc.). Publications: 30 research books, 60 book chapters, over 700 journal and conference technical papers. Editor-in Chief of the Journal of Intelligent and Robotic Systems (1988-2005) and the book series "Microprocessor-Based and Intelligent Systems Engineering" (K1uwer). Organizer of several international conferences (IEEE, IFAC, IMACS, IASTED, SIRES etc). Listed in several international biographical volumes. Current interests include: control, robotics and CIM. 